\title{
Simulation workshops as an adjunct to perioperative care bundles in the management of external ventricular drains: improving surgical technique and reducing infection
}

\author{
*Debayan Dasgupta, MBBS, MA(Cantab), ${ }^{1}$ Linda D'Antona, MBBS, ${ }^{1}$ \\ Daniel Aimone Cat, MBBS, MSc, ${ }^{1}$ Ahmed K. Toma, FRCS(SN), ${ }^{1}$ Carmel Curtis, FRCPath, PhD, ${ }^{2}$ \\ Laurence D. Watkins, FRCS(SN), ${ }^{1}$ and Lewis Thorne, FRCS(SN) ${ }^{1}$ \\ 1Victor Horsley Department of Neurosurgery, National Hospital for Neurology and Neurosurgery, Queen Square; and \\ 2Department of Clinical Microbiology, University College London Hospital NHS Foundation Trust, London, United Kingdom
}

\begin{abstract}
OBJECTIVE Temporary CSF diversion through an external ventricular drain (EVD) comes with the risk of EVD-related infections (ERIs). The incidence of ERIs varies from $0.8 \%$ to $22 \%$. ERIs increase mortality, morbidity, length of stay, and costs; require prolonged courses of antibiotics; and increase the need for subsequent permanent CSF diversion. The authors report the results of a quality improvement project designed to improve infection rates and EVD placement using simulation training in addition to a standardized perioperative care bundle. This project resulted not only in a decrease in ERIs, but also a significant improvement in surgical outcomes.
\end{abstract}

METHODS A best-practice standardized perioperative approach and care bundle was approved by consensus among the senior neurosurgeons at the authors' institution, and a standardized operative note was designed to encourage adherence to policy and improve documentation. This approach was adapted from the bundle previously described by Kubilay et al. Simulation workshops were introduced to teach safe sampling technique, administration of intrathecal drugs, and a standardized operative technique using the Rowena head surgical model. Effects of the interventions on placement, infection rates, and displacement were measured at two distinct time points over a 2-year period.

RESULTS Baseline audits demonstrated satisfactory EVD placement in $74 \%$, an infection rate of $8.5 \%$, and displacement occurring in $20 \%$. In the 2 years following the interventions, satisfactory placement improved to $96 \%$, infection rate fell to $4.8 \%$, and inadvertent displacement occurred in only $1.7 \%$.

CONCLUSIONS Simulation training and standardizing the perioperative care of patients requiring EVDs dramatically improved placement accuracy, reduced infection rates, and reduced EVD displacement rate.

https://thejns.org/doi/abs/10.3171/2018.5.JNS172881

KEYWORDS simulation training; external ventricular drains; infection; ventriculostomy; surgical technique

$\mathrm{T}$ EMPORARY CSF diversion through an external ventricular drain (EVD) is one of the most common neurosurgical procedures. Indications for placement include both as a diagnostic invasive neuromonitoring technique of intracranial pressure (ICP) in those at risk of intracranial hypertension (for example, following severe traumatic brain injury) as well as for therapeutic drainage of CSF in the presence of hydrocephalus or raised ICP.

EVD insertion comes with the risk of EVD-related infections (ERIs), commonly ventriculitis or meningitis.
Reported incidence of ERI varies in the literature from $0.8 \%$ to $22 \% .^{1,5,10}$ ERIs increase mortality, length of stay, and costs; require prolonged courses of antibiotics; and increase the need for subsequent permanent CSF diversion using an internalized shunt system, such as a ventriculoperitoneal shunt. ${ }^{4,8}$

The need for accurate EVD placement is manifold: first because the EVD must be able to effectively either monitor ICP or drain CSF, but also because incorrect placement, or multiple passes required when performing the procedure,

ABBREVIATIONS EVD = external ventricular drain; ERI = EVD-related infection; ICP = intracranial pressure.

SUBMITTED November 14, 2017. ACCEPTED May 2, 2018.

INCLUDE WHEN CITING Published online October 19, 2018; DOI: 10.3171/2018.5.JNS172881.

* D.D. and L.D. contributed equally to this work. 
have been shown to increase infection rates and morbidity. ${ }^{1,4,10}$

In response to reports of an apparent spike in ERIs at our institution from routine microbiology monitoring, a retrospective audit was initially performed to assess whether there was indeed a higher rate of infection than the published data., ${ }^{1,5,10}$ This audit demonstrated an ERI incidence of $8.5 \%(n=200$ consecutive patients at our center in 2012). This demonstrates that there was not a higher rate of infections than in previous data. However, it was noted that $70 \%$ of these infections were with gram-negative organisms, which various studies have shown lead to greater morbidity, mortality, and a longer length of stay as an inpatient. ${ }^{1}$

This was therefore followed by a prospective audit. The data from this prospective surveillance in 2013 highlighted the unexpected finding that EVD position was unsatisfactory in $26 \%$ of procedures on postoperative CT scans, with an inadvertent EVD displacement ("fall out") rate of $20 \%$. This initial prospective audit was therefore halted early, and the results triggered the stakeholder meetings that led to the design of the interventions described in this paper to both improve placement of EVDs and reduce the rate of ERIs.

We used two major interventions: the implementation of a standardized perioperative care bundle, supported by a series of advanced simulation training workshops to train the surgeons who undertake the procedure in the consensus-approved optimal method of insertion.

\section{Methods}

This quality-improvement project was registered with the Clinical Governance and Audit Department of the National Hospital for Neurology and Neurosurgery in London. The implementation of the standardized care bundle for EVD placement, perioperative management, and advanced simulation training sessions commenced in 2013. A best-practice standardized perioperative approach and care bundle was approved by consensus among the senior neurosurgeons and the lead microbiologist at the National Hospital for Neurology and Neurosurgery, and a standardized operative note was designed to improve documentation and encourage adherence to local policy. This project was adapted from the care bundle described by Kubilay et al. ${ }^{9}$

\section{Surgical Procedure}

The agreed-upon surgical procedure utilized a silver impregnated catheter; required the procedure to be performed in an approved operating theater; used prophylactic antibiotics as per hospital policy; used minimal shaving with clippers only; and involved preparation with alcoholic chlorhexidine left to air dry before draping. The procedure involves a frontal burr hole at Kocher's point, a perpendicular approach to the ventricle, ${ }^{11}$ and catheter placement at a depth of $5.5 \mathrm{~cm}$ from the cortical surface. The catheter should be tunneled for $10 \mathrm{~cm}$ and secured with an approved proprietary fixation device or using a modified Roman sandal knot. Dressing was made with three layers of permeable, transparent, water-resistant, spray-on dressing (OpSite spray, Smith \& Nephew) to the wound and exit site; no other dressings were permitted.

\section{Operative Note}

A protocol-based EVD operative note was designed to encourage adherence with policy (Fig. 1). Spaces were incorporated into the operative note to allow the surgeon to deviate from the standardized protocol and to document where and why this was done, to allow for further analysis and ongoing evolution of the optimal agreed-upon technique for the procedure.

\section{Simulation Workshops}

Simulation workshops were created to teach safe access to the CSF and administration of intraventricular drugs, and to allow surgeons to practice the standardized operative technique using the Rowena head surgical model $^{2}$ under senior observation. These workshops were each 2 hours in length and consisted of three stations. Station 1 consisted of small-group classroom teaching on indications for EVD insertion, operative technique, and peri- and postoperative management. Station 2 involved a demonstration of the optimal technique, and direct observation of the trainee's technique (by a senior neurosurgeon). Station 3 was a demonstration and observation of trainee technique of CSF sampling techniques and the delivery of intrathecal antibiotics.

Each workshop was delivered by senior neurosurgeons, senior nursing staff from the neurointensive care unit, and a senior microbiologist. Participants were given a certificate of completion and competence for their training portfolios.

The efficacy of the implemented training and care bundle was assessed in terms of reduction of ERI rates, EVD positioning, and rates of inadvertent displacement. The post-implementation prospective data collection was initially performed for 5 months, from August 2015 to January 2016. A second prospective data collection was performed for 5 months, from October 2016 to March 2017.

\section{Inclusion Criteria and Data Collection}

All adult patients (age $\geq 18$ years) undergoing EVD insertion at our neurosurgical unit were included in the study. All data were collected prospectively and included demographics, etiology, operative details, comorbidities, length of stay, morbidity and mortality, EVD position on postoperative imaging, CSF microbiology results, and antibiotic treatment. Patients were identified from the on-call neurosurgical electronic referral database and by screening the emergency and elective surgical lists. Data were derived from the patient medical case notes and electronic patient record.

The BASICS trial (British antibiotic and silver-impregnated catheters for ventriculoperitoneal shunts) definition of CSF infection was adopted for this study ${ }^{7}$ and a senior microbiologist reviewed all the CSF microbiology culture results. A summary of the criteria used for infection is the following: 1) culture positive (significant culture of organisms from the CSF); 2) organisms grown on primary culture or repeated $(>1)$ subculture; 3 ) with or without clini- 
ELECTIVE/EMERGENCY INSERTION OF EXTERNAL VENTRICULAR DRAIN (delete as appropriate)

\begin{tabular}{|c|c|}
\hline $\begin{array}{l}\text { Side: } \\
\text { Drain: }\end{array}$ & $\begin{array}{l}\text { Left/Right } \\
\text { Primary/Revision }\end{array}$ \\
\hline & $\begin{array}{l}\Gamma \text { Silver impregnated catheter } \\
\Gamma \quad \text { Other catheter (state which and reason) } \\
\end{array}$ \\
\hline Diagnosis: & $\Gamma$ SAH $Г$ Infection $\Gamma$ Tumour ${ }^{\Gamma}$ Other (please specify) \\
\hline \multirow[t]{6}{*}{ Preparation: } & $\begin{array}{l}\text { For SAH reason lumbar drain not used } \\
\Gamma \text { Procedure carried out in theatre } \bar{\Gamma} \text { Other location } \\
\text { Reason }\end{array}$ \\
\hline & $\begin{array}{l}\Gamma \text { Prophylactic antibiotics } \\
\ulcorner\quad \text { No prophylactic antibiotics (state reason) }\end{array}$ \\
\hline & $\Gamma$ Minimal Shave (CLIPPERS ONLY around entry site) \\
\hline & $\Gamma$ No shave/other shave (state which and reason) \\
\hline & $\Gamma$ Alcoholic chlorhexidine left to air dry \\
\hline & $\Gamma$ Other skin prep. (state which and reason) \\
\hline \multirow[t]{7}{*}{ Approach: } & $\begin{array}{l}\text { Frontal burr hole (mid pupillary line, behind hairline } / 2.5 \mathrm{~cm} \text { anterior to } \\
\text { Coronal suture) }\end{array}$ \\
\hline & $\Gamma$ Other location (state which and reason) \\
\hline & $\Gamma$ Catheter passed perpendicular to skull \\
\hline & $\ulcorner$ Other trajectory (state which and reason) \\
\hline & $\Gamma$ Catheter depth from surface of brain $5.5 \mathrm{~cm}$ \\
\hline & Other catheter depth (state which and reason) \\
\hline & Number of passes \\
\hline \multirow[t]{7}{*}{ Closure: } & $\Gamma$ Tunnelled $10 \mathrm{~cm}$ (measured) \\
\hline & $\Gamma$ Other length of tunnel (state which and reason) \\
\hline & $\Gamma$ Catheter secured with fixation device \\
\hline & $\ulcorner$ Catheter secured by another method (state which and reason) \\
\hline & Г Absorbable sutures to galea, staples to skin \\
\hline & $\Gamma$ Other closure (state which and reason) \\
\hline & $\begin{array}{l}\Gamma \text { Three layers of permeable, transparent, water resistant, spray on } \\
\text { dressing to wound and exit site } \\
\text { Other dressing (state which and reason) }\end{array}$ \\
\hline
\end{tabular}

FIG. 1. An example of the standardized operative note that was implemented as part of the care bundle.

cal signs of infection or malfunction; and 4) managed by shunt removal and antibiotic treatment.

EVD position on postoperative CT was classified as good, satisfactory, or unsatisfactory. Good position was when the catheter tip was surrounded by CSF in the anterior horn of the ipsilateral lateral ventricle or foramen of Monro. Satisfactory placement was when the tip was contained within the ipsilateral anterior horn of the lateral ventricle, or within the third ventricle, but the tip was abutting the ventricular wall. Any other position of the ventricular catheter was considered unsatisfactory, even if the EVD was successfully draining CSF.

\section{Statistical Analysis}

Two-tailed p values were calculated using Fisher's exact test and results were considered significant if $\mathrm{p}<0.05$.

\section{Results}

In the post-implementation period, two sets of prospective data collection of 5 months each were performed. A total of 126 EVDs were inserted in 91 patients, for which demographic data, mortality, and indications for surgery are shown in Table 1. There were no significant differences between the populations undergoing EVD place- 
TABLE 1. Demographics and indications

\begin{tabular}{|c|c|c|c|}
\hline \multirow[b]{2}{*}{ Variable } & \multicolumn{2}{|c|}{ Post-Implementation Data } & \multirow[b]{2}{*}{ Total } \\
\hline & 1st (2016) & 2nd (2017) & \\
\hline Period & Aug 2015-Jan 2016 & Oct 2016-Mar 2017 & NA \\
\hline No. of EVDs & 67 & 59 & 126 \\
\hline No. of patients & 43 & 48 & 91 \\
\hline Mean age $\pm S D$, yrs & $54 \pm 15$ & $54 \pm 18$ & $54 \pm 17$ \\
\hline Females, n (\%) & $22(51)$ & $20(42)$ & $42(46)$ \\
\hline \multicolumn{4}{|l|}{ Indications for EVD, n (\%) } \\
\hline Bleed & $37(55)$ & $32(54)$ & $69(55)$ \\
\hline Tumor & $6(9)$ & $9(15)$ & $15(12)$ \\
\hline Hydrocephalus & $10(15)$ & $9(15)$ & $19(15)$ \\
\hline Infection & $14(21)$ & $9(15)$ & $23(18)$ \\
\hline Mean drainage duration $\pm S D$, days & $9.1 \pm 5.3$ & $8.5 \pm 4.9$ & $8.8 \pm 5.1$ \\
\hline Mortality, n (\%) & $13(30)$ & $11(23)$ & $24(26)$ \\
\hline Mean hospital length of stay $\pm \mathrm{SD}$, days & $36 \pm 31$ & $23 \pm 17$ & $33 \pm 47$ \\
\hline
\end{tabular}

$\mathrm{NA}=$ not applicable.

ment between the two post-implementation data collection points in terms of their baseline demographics.

\section{EVD-Related Infections}

The rate of ERIs decreased when comparing the baseline data to the post-implementation period. This was especially true for the gram-negative microorganisms (Table 2). Although this improvement was not statistically significant $(p=0.27)$, the rate of ERIs had decreased by almost 50\%. This improvement was maintained over the 2-year period analyzed, as demonstrated in the second post-implementation data set (Table 2).

\section{EVD Position}

Postoperative scans were available for 114 patients operated on in the post-implementation period. Following simulation training, the rate of satisfactory EVD placement improved significantly, from $74 \%$ to $96 \%$ (Table 3). The improvement was statistically significant $(\mathrm{p}=0.003)$ and endured over the 2 years studied.

\section{TABLE 2. EVD-related infections}

\begin{tabular}{lcccc}
\hline & Baseline & \multicolumn{3}{c}{ Post-Implementation Data } \\
\cline { 3 - 5 } Vata & 1st & 2nd & Cumulative \\
& $(2013)$ & $(2016)$ & $(2017)$ & $(2016-2017)$ \\
\hline Total EVDs & 200 & 67 & 59 & 126 \\
\hline ERIs, $\mathrm{n}(\%)$ & $17(8.5)$ & $3(4.5)$ & $3(5.1)$ & $6(4.8)$ \\
\hline $\begin{array}{c}\text { Gram-negative infec- } \\
\text { tions, } \mathrm{n}(\%)\end{array}$ & $12(6)$ & $2(3)$ & $1(1.7)$ & $3(2.4)$ \\
\hline Comparison w/ baseline & & & & \\
\hline $\begin{array}{c}\text { ERI improvement } \\
\text { Gram-negative ERI } \\
\text { reduction }\end{array}$ & - & $p=0.42$ & $p=0.58$ & $p=0.27$ \\
\hline
\end{tabular}

\section{EVD Displacement, Standardized Operative Notes, and Length of Stay}

The latest data collection (2017) showed only 1 case of inadvertent EVD displacement over a total of 59 EVDs $(1.7 \%)$, compared to the baseline results of $20 \%(p=0.01)$. The use of the standardized EVD operative notes was $59.7 \%$. The second post-implementation data set showed a tendency toward a reduced length of stay, from a mean of 36 to 23 days, when compared to the initial post-intervention data. However, this difference was not statistically significant.

\section{Discussion}

We present the results of 126 EVDs placed in our unit over two 5-month periods across 2 years, following the implementation of a standardized perioperative care bundle and simulation training workshops. We have demonstrated a statistically significant improvement in EVD placement and a reduction in the inadvertent displacement of EVDs. There was also an apparent improvement in ERI rates and a corresponding reduction in length of stay. The reduction in ERIs did not reach statistical significance, which may reflect the relatively small numbers of infected cases in this observational study.

The improvement in placement is likely to be beneficial in terms of both morbidity and mortality. The risks of poorly positioned EVDs have been outlined in detail in previous work. ${ }^{4,5,10}$ The trend toward a reduction in ERIs, and the halving of gram-negative infections demonstrated, is consistent with previous work by Dasic et al., ${ }^{3}$ which has been replicated in subsequent studies, most notably by Flint et al. ${ }^{6}$ These authors recently published a study showing the successful reduction of their ERIs over a 4-year follow-up period using a similar protocol-based approach to infection control.

Given that the simulation training workshops and the standardized perioperative care bundle were simultane- 
TABLE 3. EVD position

\begin{tabular}{ccccc}
\hline & Baseline & \multicolumn{3}{c}{ Post-Implementation Data } \\
\cline { 3 - 5 } Vata & 1st & 2nd & \\
\hline Variable & $(2013)$ & $(2016)$ & $(2017)$ & Cumulative \\
\hline Total EVDs & 38 & 41 & 53 & 114 (12 unk) \\
\hline $\begin{array}{c}\text { Unsatisfactory EVD } \\
\text { position, } n \text { (\%) }\end{array}$ & $10(26.3)$ & $6(14.6)$ & $2(3.8)$ & $8(7)$ \\
\hline $\begin{array}{c}\text { EVD position } \\
\text { improvement }\end{array}$ & - & & & \\
\hline $\begin{array}{c}\text { Compared w/ } \\
\text { baseline }\end{array}$ & - & $p=0.26$ & $p=0.003$ & $p=0.003$ \\
\hline $\begin{array}{c}\text { Comparing } \\
\text { 2017 to 2016 }\end{array}$ & - & & $p=0.07$ & \\
\hline
\end{tabular}

Unk = unknown.

Boldface type indicates statistical significance.

ously implemented, it is impossible to distinguish directly how the addition of these workshops led to the improvements demonstrated above in catheter placement, displacement rate, and infection rates. It is possible that the formal training and observation of operative technique of the trainees, the increased awareness of the issue of ERIs in the unit, as well as an increased awareness that these were being actively monitored, all played a part in the improvements observed.

Our study supports the consensus that standardizing perioperative care bundles and improving technique reduces rates of ERIs. The study demonstrates a significant improvement in the number of procedures with satisfactory catheter placement. It also demonstrates a statistically significant reduction in the rate of inadvertent displacement of EVDs once they were inserted ("falling out"), a reduction from $20 \%$ to just $1.7 \%$. Our study shows how a relatively simple intervention can make a tangible difference to standards of care and demonstrates the added value of simulation workshops as part of this process.

\section{Conclusions}

This study adds to the growing body of evidence supporting protocol-based care bundles in the management of EVDs and demonstrates the enhanced value of additional simulation training.

\section{References}

1. Arabi Y, Memish ZA, Balkhy HH, Francis C, Ferayan A, Al Shimemeri A, et al: Ventriculostomy-associated infections: incidence and risk factors. Am J Infect Control 33:137-143, 2005

2. Ashpole RD: Introducing Rowena: a simulator for neurosurgical training. RCS Bulletin 97:299-301, 2015

3. Dasic D, Hanna SJ, Bojanic S, Kerr RSC: External ventricular drain infection: the effect of a strict protocol on infection rates and a review of the literature. Br J Neurosurg 20:296300,2006
4. Eymann R, Chehab S, Strowitzki M, Steudel WI, Kiefer M: Clinical and economic consequences of antibiotic-impregnated cerebrospinal fluid shunt catheters. J Neurosurg Pediatr 1:444-450, 2008

5. Flint AC, Rao VA, Renda NC, Faigeles BS, Lasman TE, Sheridan W: A simple protocol to prevent external ventricular drain infections. Neurosurgery 72:993-999, 2013

6. Flint AC, Toossi S, Chan SL, Rao VA, Sheridan W: A simple infection control protocol durably reduces external ventricular drain infections to near-zero levels. World Neurosurg 99:518-523, 2017

7. Jenkinson MD, Gamble C, Hartley JC, Hickey H, Hughes D, Blundell M, et al: The British antibiotic and silver-impregnated catheters for ventriculoperitoneal shunts multi-centre randomised controlled trial (the BASICS trial): study protocol. Trials 15:4, 2014

8. Keong NCH, Bulters DO, Richards HK, Farrington M, Sparrow OC, Pickard JD, et al: The SILVER (Silver Impregnated Line Versus EVD Randomized trial): a double-blind, prospective, randomized, controlled trial of an intervention to reduce the rate of external ventricular drain infection. Neurosurgery 71:394-404, 2012

9. Kubilay Z, Amini S, Fauerbach LL, Archibald L, Friedman WA, Layon AJ: Decreasing ventricular infections through the use of a ventriculostomy placement bundle: experience at a single institution. J Neurosurg 118:514-520, 2013

10. Lozier AP, Sciacca RR, Romagnoli MF, Connolly ES Jr: Ventriculostomy-related infections: a critical review of the literature. Neurosurgery 51:170-182, 2002

11. Muirhead WR, Basu S: Trajectories for frontal external ventricular drain placement: virtual cannulation of adults with acute hydrocephalus. Br J Neurosurg 26:710-716, 2012

\section{Disclosures}

The authors report no conflict of interest concerning the materials or methods used in this study or the findings specified in this paper.

\section{Author Contributions}

Conception and design: Dasgupta, D'Antona, Toma, Curtis, Watkins, Thorne. Acquisition of data: Dasgupta, D'Antona, Aimone Cat. Analysis and interpretation of data: Dasgupta, D'Antona, Aimone Cat, Curtis, Thorne. Drafting the article: Dasgupta, D'Antona. Critically revising the article: all authors. Reviewed submitted version of manuscript: all authors. Approved the final version of the manuscript on behalf of all authors: Dasgupta. Statistical analysis: Dasgupta, D'Antona, Aimone Cat. Administrative/technical/material support: Dasgupta. Study supervision: Toma, Curtis, Watkins, Thorne.

\section{Supplemental Information \\ Previous Presentations}

Portions of this work were presented in abstract and oral presentation form at the eighth meeting of the International Society for Hydrocephalus and Cerebrospinal Fluid Disorders in Cartagena, Colombia, on October 8-10, 2016.

\section{Correspondence}

Debayan Dasgupta: National Hospital for Neurology and Neurosurgery, London, United Kingdom. debayan.dasgupta1@nhs.net. 